\title{
Audible alert protected patient from inappropriate cardiac-defibrillator shocks
}

\author{
Andrzej Bissinger, Karolina Szoltek, Andrzej Lubinski \\ Department of Interventional Cardiology and Arrhythmias, \\ Medical University of Lodz, Poland
}

A 72-year-old male suffering from ischemic cardiomyopathy, left ventricular ejection fraction $30 \%$, left bundle branch block and cardiac-defibrillator (ICD) with resynchronization therapy Medtronic Cardia CRT-D since June 2013, presented to a clinic 5 days after having heard a noticeable, repeating sound from his ICD.
According to the patient's history, an alert switched on shortly after he had started using a meat grinder (Fig. 1; upper panel). Although the patient had no symptoms, the alert caused him to cease working. In effect this protected the patient from inappropriate ICD shock.

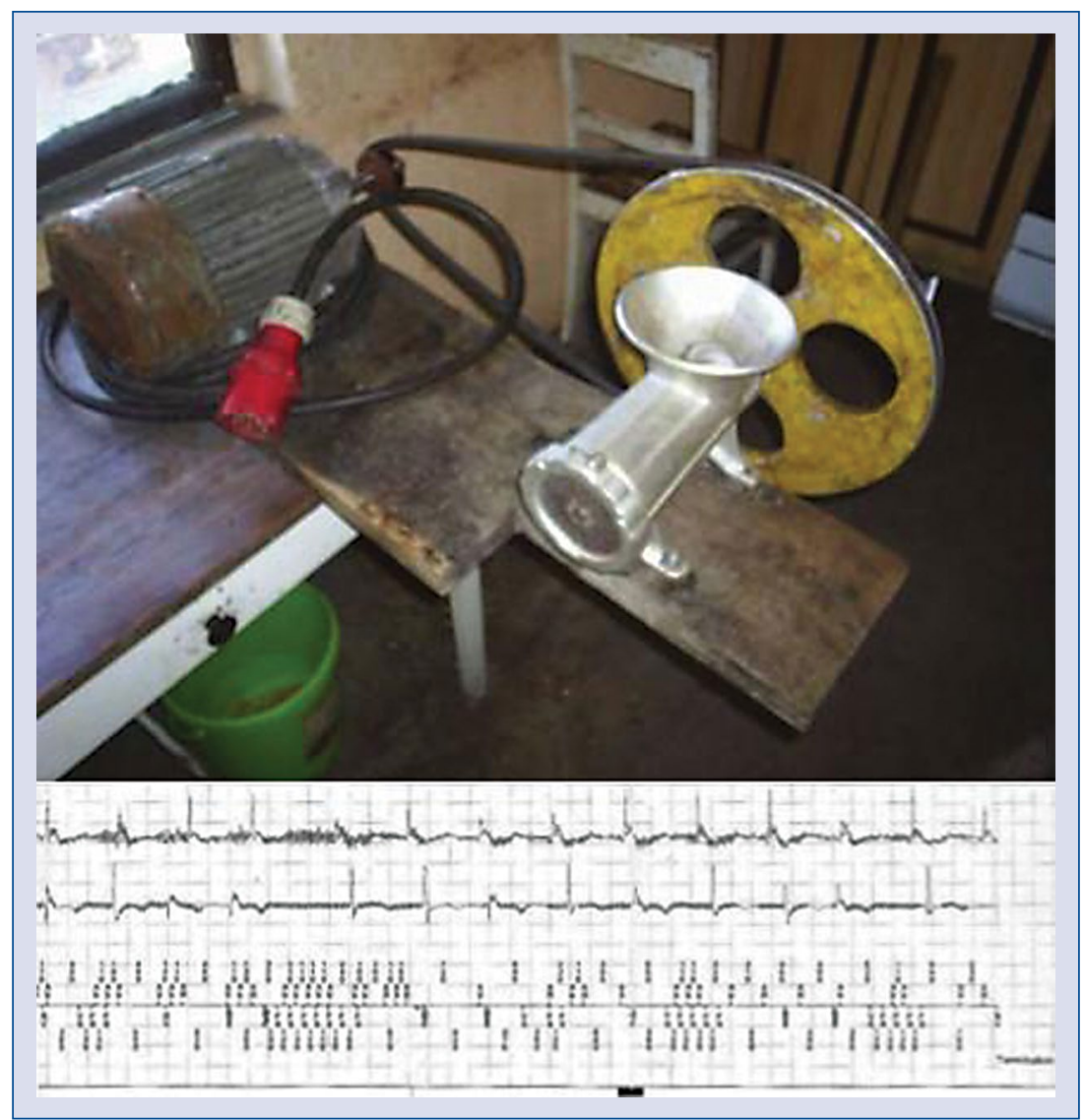

Figure 1. The meat grinder and electrograms from the implantable cardioverter-defibrillator with high frequency electricial noise detected on both — atrial and ventricular — channels.

Address for correspondence: Dr. Andrzej Bissinger, Department of Interventional Cardiology and Arrhythmias, Medical University of Lodz, ul. Żeromskiego 113, 90-549 Łódź, Poland, e-mail: abissinger@gmail.com 
While using a meat grinder, the device revealed 200 non-sustained high rate ventricular episodes which were detected as ventricular fibrillation. However, high voltage therapy was not delivered due to short single episodes. The device detected a potential lead problem by applying the right ventricle lead integrity alert (RV-LIA) algorithm, causing the device to sound the audible alarm and to temporarily suspend therapies. RV-LIA monitors RV lead impedance, frequency of rapid non-sustained ventricular tachycardia episodes with intervals $<220 \mathrm{~ms}$ and the frequency of short ventricular intervals $(\mathrm{V}-\mathrm{V}<130 \mathrm{~ms})$. Meanwhile intracardiac electrograms showed high frequency microvoltages-bursts on both channels of ICD (Fig. 1).

Despite high effectiveness and safety of ICD, it had a weakness. It may provide inappropriate shocks. Electromagnetic interference is a rare cause, nevertheless this case has shown a significant one. Contemporary algorithms make programming changes to protect patients in such situations. Also once the RV-LIA criteria are met, an alert occurs immediately. In conclusion it was not possible to predict all electromagnetic interference with ICD. Patients should therefore be instructed to investigate all audible alarms from their device.

Conflict of interest: None declared 